\title{
Preface for the special issue on Ontologies and Logic Programming for Query Answering
}

\author{
Odile Papini $^{1} \cdot$ Salem Benferhat ${ }^{2}$
}

Published online: 29 March 2018

(C) Springer International Publishing AG, part of Springer Nature 2018

This special issue collects fully revised and extended versions of contributions presented at the international workshop Ontologies and Logic Programming for Query Answering (ONTOLP 2015) affiliated with the 24th International Joint Conference on Artificial Intelligence (IJCAI-2015) which took place in Buenos Aires, Argentina, July 25th, 2015 in the "Facultad de Ciencias Economicas" at the University of Buenos Aires.

The aim of this workshop was to bridge knowledge representation and reasoning in artificial intelligence and web of knowledge communities in order to encourage the emergence of new solutions for reasoning with lightweight ontologies. The workshop focused on languages and techniques that allow for:

- Query answering while taking ontologies into account.

- Nonmonotonic reasoning that deals with inconsistencies and exceptions in ontologies.

Concerning the first point, a challenging issue was how to adapt or extend Answer Set Programming (ASP) to represent ontological knowledge. In particular, can (a fragment of) ASP cover lightweight ontological languages while keeping decidability and efficiency?

Concerning the second point, a challenging issue was how to extend lightweight ontological languages with non-monotonic features, without increasing computational complexity of query answering. In particular,

i) how to embed exceptions-based and inconsistency tolerant-based reasoning in a tractable ontological language?

ii) how to integrate uncertainty information in lightweight ontological languages?

Odile Papini

odile.papini@univ-amu.fr

Salem Benferhat

benferhat@cril.univ-artois.fr

1 LIS-CNRS UMR 7020, Aix-Marseille Université, Campus de Luminy, Marseille, France

2 CRIL-CNRS UMR 8188, Université d'Artois, Faculté Jean Perrin, Lens, France 
iii) how to define merging operations where both inputs and outputs are in lightweight ontological languages?

The first paper "Bringing existential variables in answer set programming and bringing nonmonotony in existential rules: two sides of the same coin" by Jean-François Baget, Laurent Garcia, Fabien Garreau Claire Lefèvre, Swan Rocher and Igor Stéphan, proposes a new framework (called ENM-rules) which both integrates ontologies and rules in a unique formalism. The paper first proposes a definition of an ASP with existential variables. The treatment of these variables is done in terms of skolemization. Then it expands the standard existential rules formalism (also called Datalog+/-) which generalizes lightweight description logics such as DL-Lite and EL, with a nonmonotonic negation under stable model semantics.

The next paper "How does Incoherence affect Inconsistency-tolerant Semantics for Datalog" by Cristhian Ariel David Deagustini, M. Vanina Martinez, Marcelo Alejandro Falappa and Guillermo Ricardo Simari, addresses a problem that is important for answering queries from inconsistent knowledge bases in semantic web applications. The authors consider scenarios where different sources of information need to be integrated, where such integration is prone not only to errors at the database level but also at the constraints one. The paper proposes a new reasoning approach in presence of both incoherence and inconsistency with an alternative semantics stemming on argumentative inference.

The third paper "Upward Refinement Operators for Conceptual Blending in the Description Logic EL++" by Roberto Confalonieri, Manfred Eppe, Marco Schorlemmer, Oliver Kutz, Rafael Peñaloza and Enric Plaza, addresses generalisation within the framework of EL++. It presents a formalisation of the generalisation process that relies on the notion of refinement operators defined in Inductive Logic programming and on the notion of amalgation developped in case-based reasoning. Refinement operators are defined for EL++ and their properties are studied. An ASP implementation is provided for computing the generalized EL++ concepts and an algorithm is given for blending the generalized EL++ concept.

In the literature, tableau algorithms are often used to answer querying Description Logics knowledge bases tableau algorithms. The fourth paper "Tableau Reasoning for Description Logics and its Extension to Probabilities" by Riccardo Zese, Elena Bellodi, Fabrizio Riguzzi, Giuseppe Cota and Evelina Lamma, presents two tableau reasoners TRILL for ALC knowledge bases and TRILL ${ }^{P}$ for SHOIQ knowledge bases that implement in PROLOG a tableau algorithm with the ability of returning explanations and managing probabilistic answer querying.

The fifth paper "Quasi-classical Reasoning in Paraconsistent Databases" by Badrinath Jayakumar and Rajshekhar Sunderraman, proposes a paraconsistent relational model in order to represent negative information in deductive databases based on quasi-classical logic (QC), a four valued logic close to classical logic, to support disjunctive information.

The last paper "Parsing as Semantically Guided Constraint Solving: the Role of Ontologies" by Veronica Dahl, Sergio Tessaris and Mariano De Sousa Bispo, is in the framework of natural language processing. The paper extends the parsing as constraint model with semantic information represented by ontologies.

We would like to thank the ANR ASPIQ (project reference ANR-12-BS02-0003) for its support to the workshop. We are most grateful to the following referees for their help and valuable works during the reviewing process: Mario Alviano, Jean-François Baget, Maria Teresa Alsinet Bernado, Madalina Croitoru, James Delgrande, Thomas Eiter, Jérôme Euzenat, Eduardo Fermè, Sergio Alejandro Gomez, Ricardo Gonçalves, Laurent Garcia, Fabio Grandi, Gabriele Kern-Isberner, Sylvain Lagrue, Claire Lefèvre, Thomas Lukasiewicz, Stéphane Loiseau, Robert E. Mercer, Michael Morak, Thomas Meyer, Farid Nouioua, Marie-Christine Rousset, Torsten Schaub, Igor Stéphan, Antonius Weinzierl, Eric Würbel. 